\title{
Impact of Pathogen-Specific Antibiotic on Clinical Outcomes in Ventilator-Associated Pneumonia
}

\author{
Isra Halim¹, Kiran Chawla², Muralidhar Varma ${ }^{3}$ \\ ${ }^{1,2}$ Department of Microbiology, Kasturba Medical College, Manipal, Karnataka, India. ${ }^{3}$ Department of \\ Medicine, Kasturba Medical College, Manipal, Karnataka, India.
}

\section{ABSTRACT}

\section{BACKGROUND}

Ventilator-associated pneumonia (VAP) is a prime cause of morbidity and mortality in intensive care settings. Institution of specific antibiotic therapy in VAP could be instrumental in reducing intensive care unit (ICU) utilisation rates in terms of days on ventilator and days admitted besides improving patient outcomes.

\section{METHODS}

In a prospective study on 100 VAP patients, endotracheal aspirates from cases were processed microbiologically according to standard procedures. Patients in whom empiric antibiotics were modified to pathogen-specific antibiotics following susceptibility report, were compared with the group where pathogen-specific antibiotics were not administered. Outcomes were measured in terms of duration of mechanical ventilation, length of ICU stay and mortality rates in both study groups.

\section{RESULTS}

Among the 100 study cases, $84 \%$ were changed to pathogen-specific antibiotic therapy in our ICU. The average duration of mechanical ventilation was comparable in both groups, 7.5 days in patients on administered targeted therapy, as compared to 8 days in patients whose antibiotics were not changed. Similarly, mean length of ICU stay was marginally shorter (10 days) in the former group of patients as compared to the latter (10.5 days). Mortality rates were significantly associated with a change in antibiotic therapy. A staggering $50 \%$ patients belonging to the noncompliant group died in the ICU, as opposed to $38.4 \%$ in the group whose antibiotics were changed $(\mathrm{P}<0.05)$.

\section{CONCLUSIONS}

Therapy in VAP should be modified according to microbiological susceptibility reports so as to shorten days in ICU, shorten the duration on invasive ventilation and bring down mortality rates in VAP.

\section{KEY WORDS}

Ventilator-Associated Pneumonia, Antibiotic Therapy
Corresponding Author: Dr. Isra Halim, 298 Patliputra Colony, Patna - 800013, Bihar, India. E-mail: israhalim@gmail.com

DOI: $10.14260 /$ jemds/2021/40

How to Cite This Article:

Halim I, Chawla K, Varma M. Impact of pathogen-specific antibiotic on clinical outcomes in ventilator-associated pneumonia. J Evolution Med Dent Sci 2021;10(04):185-189, DOI: $10.14260 / \mathrm{jemds} / 2021 / 40$

Submission 16-09-2020, Peer Review 24-11-2020, Acceptance 30-11-2020, Published 25-01-2021.

Copyright (c) 2021 Isra Halim et al. This is an open access article distributed under Creative Commons Attribution License [Attribution 4.0 International (CC BY 4.0)] 


\section{BACKGROUND}

Mechanical ventilation has been historically proven to increase the risk of pneumonia in intensive care settings. Ventilator associated pneumonia is defined as a pneumonia where the patient is on mechanical ventilation for more than 2 calendar days on the date of the event, with the day of ventilator placement being day 1 and the ventilator was in place on the date of the event or the day before (Centers for Disease Control and Prevention). ${ }^{1}$ Mortality rates attributable to VAP computed across various settings have been as high as $76 \% .^{2-4}$ Although host factors and underlying premorbidities play a decisive role in patient outcomes, appropriate antibiotic regimes-constituting the right drug, in the right dose, for the right duration of treatment could possibly lead to superior patient outcomes. Studies across the world have reported an unfavourable clinical outcome in patients administered inappropriate empirical therapy in VAP.5-8 Inappropriate empirical antibiotic regimens have strongly been associated with increased length of hospital stay, morbidity and mortality, besides encouraging the dreaded problem of emerging drug resistance.6,9 However, the role of rapid microbiological diagnosis and institution of appropriate, i.e. pathogen-specific antibiotics following susceptibility testing in curtailing the duration on mechanical ventilation, length of intensive-care unit stay and preventing further mortality is yet understated. The question whether susceptibility reports are an effective guide for clinicians and intensivists in improving patient outcomes in VAP is still unanswered. Does in vitro susceptibility testing for bacterial isolates from VAP essentially translate into improved end results of antibiotic therapy in these critically ill patients? Could optimum and appropriate pathogen-specific antibiotic therapy contribute in reducing costs of healthcare by decreasing ventilator and inICU days? Available data pertaining to these perturbing questions are scarce and fragmentary, in our settings. This study attempts to inspect the effect of targeted therapy, according to microbiological report, in terms of clinical outcomes among VAP patients.

\section{METHODS}

A prospective observational study was conducted in two central ICU's of a tertiary care university hospital in South India. Laboratory workup was performed in the Department of Microbiology. The study spanned a duration of 18 months, from September 2013 to March 2015.

A sample size of 93 was obtained statistically based on the estimation of proportions. For analytical convenience, 100 non-consecutive adult patients, mechanically ventilated for more than 48 hours, fulfilling the Clinical Pulmonary Infection Score (CPIS) criteria were included in the study. ${ }^{10}$ Paediatric patients and those presenting with pneumonia on admission were excluded.

Ethics approval was obtained from the institutional ethics committee before the start of the study. Informed consent from patients' next of kin was taken.

\section{Microbiological Workup}

Endotracheal aspirate from included patients with suspected VAP were collected by trained respiratory therapists in sterile conditions using a 22-inch Romson's ${ }^{\circledR} 12$ F suction catheter equipped with a mucus extractor. The sample was sent to the microbiology laboratory for processing within 30 minutes of collection.

\section{Staining and Aerobic Culture}

A Gram stain was performed on the sample, to look for inflammatory cells and bacteria. Samples with $\leq 5$ epithelial cells / low power field and $\geq 25$ pus cells / low power field were included for further processing. For aerobic culture, the specimens were cultured semi-quantitatively as per standard guidelines. Samples were plated on $5 \%$ sheep blood Agar, MacConkey's Agar and Chocolate Agar.

While the blood agar and chocolate agar plates were incubated at $37^{\circ} \mathrm{C}$ in $5 \% \mathrm{CO} 2$ incubator for 24 hours, the MacConkey's Agar plate was incubated at the same temperature in an ambient air incubator. Colony counts of $\geq$ 105 colony forming units / $\mathrm{mL}$ (cfu / $\mathrm{mL}$ ) were considered to be suggestive of VAP. Those less than this cut-off were considered either colonizers or contaminants. The isolates were identified on the basis of gram stain, colony characteristics and a battery of basic biochemical tests.

\section{Antimicrobial Susceptibility Testing}

The antimicrobial susceptibility was performed by the Kirby Bauer's disc diffusion method on Mueller-Hinton agar. It was interpreted in accordance with the Clinical Laboratory Standard Institute (CLSI), 2013 guidelines.

\section{Antimicrobial Therapy}

In patients suspected of developing VAP, initial antibiotic therapy was started after taking preliminary samples for microbiological analysis. Initial therapy was based on the discretion of the consultant physician based on the clinical presentation, co-morbidities, length of hospitalisation and local epidemiology and susceptibilities. The decision to continue or change empiric therapy according to the microbiological susceptibility report was also left to the physician's discretion. The consulting physician was blind to this study to avoid bias in therapeutic approach.

\section{Demographic Data Collection}

Clinico-epidemiological variables such as age, gender, diagnosis at admission, history of prior trauma or surgery, chronic obstructive pulmonary disease (COPD) and septic shock were recorded from clinical case sheets.

\section{Follow-Up of the Study Cohort}

Patients enrolled were followed up daily until death or discharge. The duration of mechanical ventilation and total length of ICU stay was recorded for all patients included in the study. Both clinical improvement and deterioration-in terms of resolution of symptoms, radiological improvement and normalizing leucocyte counts along with endotracheal culture 
growth every 72 hours-were monitored to assess the antibiotic response in the study cases.

\section{Statistical Analysis}

Clinical, epidemiological and microbiological data was analyzed using SPSS for Windows, Version 16.0. Chicago, SPSS Inc. Software. Descriptive statistics were employed to estimate frequencies and proportions. Contingency tables measuring outcomes were analysed using the chi-square test. Nonparametric Mann-Whitney U-test was used in case of continuous variables. Differences between groups were considered to be significant for variables yielding a P-value < 0.05 .

\section{Definitions}

\section{Early Onset VAP}

VAP occurring within the first 4 days of mechanical ventilation. ${ }^{11}$

\section{Late Onset VAP}

VAP occurring on or after 5 days of mechanical ventilation. ${ }^{11}$

\section{Empiric Treatment}

Antimicrobials prescribed for fever or other systemic signs of infection (eg. hypothermia, leukocytosis) without identifying a specific localized source of infection.

\section{Pathogen Specific Antibiotic}

Antimicrobials prescribed for a proven pathogen according to microbiological susceptibility reports.

\section{Response to Therapy}

Patients were deemed clinically improved if fever disappeared and if pulmonary infiltrates and physical signs of pneumonia abated.

\section{Multi Drug Resistant (MDR)}

Acquired non-susceptibility to at least one agent in three or more antimicrobial categories. ${ }^{13}$

\section{Extensively Drug Resistant (XDR)}

Non-susceptibility to at least one agent in all but two or fewer antimicrobial categories (i.e. bacterial isolates remain susceptible to only one or two categories). ${ }^{12}$

\section{RESULTS}

During the study 100 cases of ventilator associated pneumonia were included. A total of 128 bacteria was isolated from these cases.

\section{Clinico-Epidemiological Characteristics of Study Cases}

The study population included adults between the ages of 18 85 years. The mean age of the population was 50.11 (SD \pm 17.96). Our study cohort had a male preponderance with a male to female ratio of 2.8:1.
The median duration of ICU stay for our study population was 10 days (interquartile range-IQR $7,15.75$ ) days. On an average, each patient was ventilated for 7.78 days with a standard deviation of 3.32 days. In terms of condition at discharge, $47 \%$ of the total patients improved clinically. On the other hand, $40 \%$ died in hospital. Further details are tabulated as follows. (Table I)

A majority of our study cases were admitted with cardiorespiratory symptoms $(24 \%)$. The study population also comprised of patients with cardiovascular symptoms, trauma and poisoning the details of which are enlisted in Table II.

In our study, VAP occurred within 96 hours of mechanical ventilation, i.e. early onset in 54 of 100 cases. The remaining 46 patients contracted VAP beyond 96 hours of intubation.

\section{Microbiological Profile of Isolates}

From a total of 100 study cases, 128 pathogens were isolated. of the total isolates, $83(64.9 \%)$ were multidrug resistant (MDR) strains.

Microbiologically, 115 (89.8 \%) gram negative isolates and 13 gram positive isolates were obtained. Among gram negative isolates, non-fermenters including acinetobacter spp, pseudomonas spp and Stenotrophomonas maltophilia comprised a majority of the total pathogens isolated (63\%). $95.6 \%$ of the total acinetobacter strains isolated were found to be MDR, as against $56.2 \%$ klebsiella and $53.5 \%$ pseudomonas strains. Of note was the increasing incidence of extensively drug-resistant XDR gram negative bacteria isolated. 74 of the gram negative rods isolated in the study were extensively drug resistant; i.e. showing resistance to all classes of antibiotics tested, with the exception of colistin and tigecycline (64.3\%).

Staphylococcus aureus was the predominant organism among gram positive isolates comprising 10 out of the 13gram positive VAP cases (76.92\%). Staphylococcus aureus were found to be methicillin resistant strains (MRSA) in 8 cases of staphylococcal VAP (80\%).

Comparison of the patient group with no change in therapy vs. those with change in antibiotic therapy with respect to the length of ICU stay, duration of mechanical ventilation and mortality.

Among the 100 study cases, 84 were switched to pathogen specific antibiotic in compliance with microbiological susceptibility reports. 16 patients were continued on empirical antibiotic therapy.

The impact of change of antibiotic therapy following microbiological report was measured in terms of duration of mechanical ventilation, length of stay in the ICU and clinical outcome.

While a change or de-escalation in antibiotic therapy according to microbiological report did not considerably decrease the duration on mechanical ventilation or the length of stay in ICU for our study cases, it significantly affected their clinical outcomes. It was observed that the group that changed to pathogen specific antibiotic therapy showed survival rates of $51.2 \%$, as against only $21.4 \%$ survival in the group that was continued on empiric antibiotic alone. Death in hospital was recorded in half of the patients whose antibiotic was not changed, while mortality in the group whose antibiotics were changed was $38.4 \%$. (Table 3 ). 


\begin{tabular}{|cc|}
\hline Patient & $\mathbf{N}=\mathbf{1 0 0}$ \\
Age (Range) & $18-85$ \\
Mean & $50.11 \pm 17.96$ \\
Gender & \\
Male & 74 \\
Female & 26 \\
Duration of ICU stay (Range) & $3-46$ days \\
Median duration (IQ3- IQ1) & $10(7.0-15.75)$ \\
Duration of mechanical ventilation (Range) & $3-16$ days \\
Mean duration \pm SD & $7.78 \pm 3.32$ \\
Clinical outcome & $47 \%$ \\
Clinical improvement & $40 \%$ \\
Total number of deaths in hospital & $13 \%$ \\
Clinical worsening (DAMA) & \\
\hline Table 1. Clinico-Epidemiologic Characteristics of Cases \\
\hline
\end{tabular}

\begin{tabular}{|cc|}
\hline Underlying Diseases & Frequency (n) \\
\hline Shock / MODS & 18 \\
CNS & 16 \\
RS & 15 \\
CVS & 9 \\
Trauma & 8 \\
Poisoning & 7 \\
Gastrointestinal tract & 6 \\
Malignancy & 6 \\
Post-surgery & 4 \\
Others & 11 \\
\hline Table 2. Underlying Disease at Presentation $(\mathbf{N}=\mathbf{1 0 0})$ \\
\hline
\end{tabular}

\begin{tabular}{|cccc|}
\hline & $\begin{array}{c}\text { Pathogen Specific } \\
\text { Antibiotics } \\
\text { Changed (N = 84) }\end{array}$ & $\begin{array}{c}\text { Pathogen Specific } \\
\text { Antibiotics Not } \\
\text { Changed (N = 16) }\end{array}$ & P Value \\
\hline $\begin{array}{c}\text { Duration of mechanical } \\
\text { ventilation }\end{array}$ & $7.5(5.0-9.25)$ & $8(4.0-10.25)$ & 0.95 \\
Length of ICU stay & $10(7-15)$ & $10.5(6.5-20.5)$ & 0.81 \\
\hline \multicolumn{3}{|c|}{ Clinical Outcome } \\
\hline Dearvival & $44(51.2 \%)$ & $3(21.4 \%)$ & $0.05^{*}$ \\
\hline Deterioration & $33(38.4 \%)$ & $7(50 \%)$ & \\
\hline \multicolumn{2}{|c|}{ Table 3. Outcome Assessment with Change to } \\
Pathogen Specific Therapy in VAP \\
\hline *Mann-Whitney U test, **Chi square test \\
\hline
\end{tabular}

\section{DISCUSSION}

Empirical antibiotic therapy entails the initial selection of an antibiotic regimen that aims to be effective against any pathogen suspected of causing the infection. ${ }^{13}$ Based on the local microbial prevalence in the ICU, most patients are started empirically on broad spectrum antibiotics. The most frequent drug administered as initial therapy in our settings are betalactam (BL) + beta-lactamase inhibitor (BLI) combinations like piperacillin-tazobactam and cefoperazone-sulbactam. However, de-escalation of the initially commenced broadspectrum therapy, has been advocated based on antibiotic susceptibility testing. ${ }^{14}$ Microbiological information has been proposed as a useful tool for modifying initial empiric treatment allowing a more targeted and cost-effective therapy. ${ }^{7,15}$

Among the 100 cases in our study, 84 were administered pathogen specific antibiotic therapy according to microbiology report. 16 patients were however, not given appropriate antibiotic therapy. Researchers in the past two decades have reported comparatively lower rates of administration of appropriate antibiotic therapy in VAP patients ranging between 25 to $66 \%$ in different settings. ${ }^{7,16}$ Data from a tertiary care centre in India reports up to one-third of their
VAP patients not being treated appropriately according to microbiological susceptibility reports. ${ }^{16}$ The gradual increase in the use of targeted therapy over time points towards a corresponding increase in compliance to microbiological susceptibility reports across the world. However, the reasons for not replacing empiric therapy by specific therapy in our settings could be diverse. Firstly, many of our patients presented with VAP due to extensively drug resistant pathogens sensitive only to colistin. Colistin could not be administered in some of these critically ill patents owing to impaired renal parameters. Unfortunately, a small proportion of patients were also unable to bear the costs of treatment with these expensive antibiotics. Moreover, "collateral damage" due to de-escalation of some antibiotics like cephalosporins in selecting drug resistant variants could also have contributed to the physician's decision against downscaling empiric therapy. ${ }^{17}$

VAP patients in our ICU had a median duration of stay of 10 days (IQR 7, 15.7 days). This was in concordance with a study done by Rello and coworkers, ${ }^{18}$ who reported a significant increase in the duration of ICU stay (11.7 days \pm 11 days) among VAP patients as opposed to non-VAP patients in ICU. Similar results were reported by Arvanitis et al. ${ }^{19,20}$ This prolonged duration of ICU stay is not attributable to the underlying disease condition alone. Since, most of these patients have usually been treated in peripheral centres before presentation to our hospital, it is likely that they have been treated with antibiotics. Prior antibiotic therapy has long been known as a risk factor for the selection of multi drug resistant bacteria. These infections, being more resistant to therapy with commonly used antibiotics result in prolonged spells of ICU stay.

Our cases were mechanically ventilated for a mean duration of $7.78 \pm 3.32$ days. Studies on VAP have reported a slightly higher duration of mechanical ventilation ranging between 9 - 20 days. This could possibly be explained by the higher proportion of cases of early onset VAP in our study, which are known to be easier to treat than late onset VAP.

Both the duration of mechanical ventilation and the length of stay in ICU were more in the patient group whose antibiotics were not changed according to reports. However, statistical significance was not reached probably due to a small sample size. Data comparing clinical outcome in these two groups is unfortunately sparse.

Furthermore, most of the patients on inappropriate therapy were found to be at a significantly higher risk for death ( $\mathrm{p}<0.05$ ). This is in concordance with many studies in the past. 16,18,21,22 In our study, a significant half of the group in whom antibiotic therapy was not modified according to reports succumbed to in-ICU mortality. This is in contrast with findings of Rello et al. who reported an additional ICU mortality rate of merely $14.4 \%$ in VAP patients treated inappropriately. ${ }^{18} \mathrm{~A}$ smaller comparison group of patients in our study could be accountable for this disparity. Unfavourable host factors and primary condition on admission could also have contributed to higher mortality rates in our study.

A major strength of our study was that all study cases were followed up clinically and microbiologically until improvement, death or discharge. Nonetheless, for ethical reasons, our study design was strictly observational. Possible confounders such as host factors could not be eliminated. Also, 
due to the non-consecutive selection of cases, a possibility of a bias cannot be ignored. Thirdly, information on previous recent antibiotic exposure was not collected. This could have further strengthened the association between multi drug resistant organisms and treatment outcomes in VAP. Further time-bound studies, with larger population groups could more accurately define the effect that pathogen specific antibiotic therapy has no treatment outcomes in VAP.

Despite these limitations, appropriate antibiotic therapy tailored according to microbiology reports in VAP patients is associated with definite survival advantages in our settings. A shorter duration of mechanical ventilation and shorter length of ICU stay as a result of appropriate pathogen specific therapy would also go a long way in improving patient outcomes, decreasing additional resource utilisation in the ICU and reducing costs of healthcare amongst this critical group of patients.

\section{CONCLUSIONS}

Microbiological susceptibility reports remain a corner stone in reducing the days in ICU, duration on mechanical ventilation and can help bring down mortality rates in VAP considerably. Clinicians must de-escalate therapy in VAP in accordance with these reports. A shorter duration of mechanical ventilation and shorter length of ICU stay as a result of appropriate pathogen specific therapy would also go a long way in improving patient outcomes, decreasing additional resource utilisation in the ICU, and reducing costs of healthcare amongst this critical group of patients.

Data sharing statement provided by the authors is available with the full text of this article at jemds.com.

Financial or other competing interests: None.

Disclosure forms provided by the authors are available with the full text of this article at jemds.com.

\section{REFERENCES}

[1] CDC. Pneumonia (Ventilator-associated [VAP] and nonventilator-associated Pneumonia [PNEU]) Event. 2021.

http://www.cdc.gov/nhsn/pdfs/pscmanual/6pscvapcur rent.pdf.

[2] Ranjan N, Chaudhary U, Chaudhry D, et al. Ventilatorassociated pneumonia in a tertiary care intensive care unit: analysis of incidence, risk factors and mortality. Indian J Crit Care Med 2014;18(4):200-4.

[3] Chastre J, Fagon JY. Ventilator-associated pneumonia. Am J Respir Crit Care Med 2002;165(7):867-903.

[4] Koenig SM, Truwit JD. Ventilator-associated pneumonia: diagnosis, treatment and prevention. Clin Microbiol Rev 2006;19(4):637-57.

[5] Kollef MH, Sherman G, Ward S, et al. Inadequate antimicrobial treatment of infections: a risk factor for hospital mortality among critically ill patients. Chest 1999;115(2):462-74.

[6] Luna CM, Vujacich P, Niederman MS, et al. Impact of BAL data on the therapy and outcome of ventilator-associated pneumonia. Chest 1997;111(3):676-85.
[7] Rello J, Gallego M, Mariscal D, et al. The value of routine microbial investigation in ventilator-associated pneumonia. Am J Respir Crit Care Med 1997;156(1):196200.

[8] Kollef MH, Ward S. The influence of mini-BAL cultures on patient outcomes: implications for the antibiotic management of ventilator-associated pneumonia. Chest 1998;113(2):412-20.

[9] Gadani H, Vyas A, Kar AK. A study of ventilator-associated pneumonia: incidence, outcome, risk factors and measures to be taken for prevention. Indian J Anaesth 2010;54(6):535-40.

[10] Pugin J. Clinical signs and scores for the diagnosis of ventilator-associated pneumonia. Minerva Anestesiol 2002;68(4):261-5.

[11] American Throacic Society, Infectious Diseases Society of America. Guidelines for the management of adults with hospital-acquired, ventilator-associated and healthcareassociated pneumonia. Am J Respir Crit Care Med 2005;171(4):388-416.

[12] Magiorakos AP, Srinivasan A, Carey RB, et al. Multidrugresistant, extensively drug-resistant and pandrugresistant bacteria: an international expert proposal for interim standard definitions for acquired resistance. Clin Microbiol Infect 2012;18(3):268-81.

[13] Swanson JM, Wells DL. Empirical antibiotic therapy for ventilator-associated pneumonia. Antibiotics (Basel) 2013;2(3):339-51.

[14] Paterson DL. Impact of antibiotic resistance in gramnegative bacilli on empirical and definitive antibiotic therapy. Clin Infect Dis 2008;47(Suppl 1):S14-20.

[15] Rello J, Ollendorf DA, Oster G, et al. Epidemiology and outcomes of ventilator-associated pneumonia in a large US database. Chest 2002;122(6):2115-21.

[16] Joseph NM, Sistla S, Dutta TK, et al. Outcome of ventilatorassociated pneumonia: impact of antibiotic therapy and other factors. Australas Med J 2012;5(2):135-40.

[17] Paterson DL. Collateral damage from cephalosporin or quinolone antibiotic therapy. Clin Infect Dis 2004;38(Suppl 4):S341-5.

[18] Rello J, Vidaur L, Sandiumenge A, et al. De-escalation therapy in ventilator-associated pneumonia. Crit Care Med 2004;32(11):2183-90.

[19] Arvanitis M, Anagnostou T, Kourkoumpetis TK, et al. The impact of antimicrobial resistance and aging in VAP outcomes: experience from a large tertiary care center. PLoS One 2014;9(2):e89984.

[20] El-Saed A, Balkhy HH, Al-Dorzi HM, et al. Acinetobacter is the most common pathogen associated with late-onset and recurrent ventilator-associated pneumonia in an adult intensive care unit in Saudi Arabia. Int J Infect Dis 2013;17(9):e696-e701.

[21] Höffken G, Niederman MS. Nosocomial pneumonia: the importance of a de-escalating strategy for antibiotic treatment of pneumonia in the ICU. Chest 2002;122(6):2183-96.

[22] Richards MJ, Edwards JR, Culver DH, et al. Nosocomial infections in medical intensive care units in the United States. Crit Care Med 1999;27(5):887-92. 